Research Paper

\title{
Clinical Effects of Xinmailong Therapy in Patients with Chronic Heart Failure
}

\author{
Qilin Ma², Youxi Luo', Pi Guo1,3, Gan Gao², Meixue Yang1, Gaurav Sablok, Yanchun Zhang5,6, Fengfeng \\ $\mathrm{Zhou}^{1, \mathbb{}}$,
}

1. Shenzhen Institutes of Advanced Technology, and Key Laboratory of Health Informatics, Chinese Academy of Sciences, Shenzhen, Guangdong, P.R. China, 518055;

2. Cardiovascular Internal Department, Xiangya Hospital, Central South University, Changsha, Hunan, P.R. China, 410008;

3. Department of Public Health, Shantou University Medical College, No. 22 Xinling Road, Shantou, Guangdong, P.R. China, 515041;

4. Department of Biodiversity and Molecular Ecology, Research and Innovation Centre, Fondazione Edmund Mach, Via E. Mach 1, 38010 S. Michele all'Adige (TN), Italy.

5. Centre for Applied Informatics, College of Engineering and Science, Victoria University, PO Box 14428, Melbourne, VIC 8001, Australia.

6. Centre for Ficticious Economy and Data Science, University of Chinese Academy of Science. \#305, Building 6, Zhongguancun East Road 80, Haidian District, Beijing, China 100190.

$\bowtie \quad$ Corresponding author: Fengfeng Zhou, Email: ff.zhou@siat.ac.cn or FengfengZhou@gmail.com. Web site: http://www.HealthInformaticsLab.org/ffzhou/.

( ) Ivyspring International Publisher. This is an open-access article distributed under the terms of the Creative Commons License (http://creativecommons.org/ licenses/by-nc-nd/3.0/). Reproduction is permitted for personal, noncommercial use, provided that the article is in whole, unmodified, and properly cited.

Received: 2012.12.29; Accepted: 2013.03.II; Published: 2013.03 .23

\begin{abstract}
In the last 100 years, intensive studies have been done on the identification of the systematic approaches to find the cure for the chronic heart failure, however the mystery remains unresolved due to its complicated pathogenesis and ineffective early diagnosis. The present investigation was aimed to evaluate the potential effects of the traditional chinese medicine, Xinmailong, on the chronic heart failure (CHF) patients as compared to the standard western medical treatment available so far. In our study, we selected two groups of voluntary CHF patients at the Xiangya Hospital, which were allowed to administrate Xinmailong or standard treatments, respectively. Another group of voluntary healthy individuals were recruited as the control group. The treatment effectiveness was measured by five symptomatic factors, i.e. angiotensin II (Ang_II), high sensitivity C-reactive protein (hs_CRP), Left Ventricular End Systolic Volume Index (LVESVI), left ventricular ejection fraction (LVEF) and pro-B-type natriuretic peptide (NT_proBNP), between the control group and the CHF patients at different stages of drug administration and in different treatment groups. The timeline for the full dose administration was set to 15 days and five measurements as indicated above were taken on every 0,7 th and 15 th day of the drug administration respectively. In the conducted study, similar symptomatic measurements were observed on day 0 in both treatment groups, and slight improvements were observed on 7th day. It was observed that after a full course of drug administration for 15 days, both of the treatment groups achieved statistically significant improvements in all the five measures, but Xinmailong was found to be more (almost double) statistically significant as compared with the available drug treatments for chronic heart failure.
\end{abstract}

Key words: Chronic heart failure, Traditional Chinese Medicine, Xinmailong.

\section{Introduction}

Heart failure, a common disorder, is due to impaired heart pumping ability, and the chronic heart failure $(\mathrm{CHF})$ is usually induced by the damaged structure or function of the left ventricle [1, 2]. Although CHF may occur at any age, it is most commonly observed in patients of 76 or older [3]. It has 
been earlier reported that the ejection efficiency of left ventricle may be impaired by several diverse factors, which includes aging, diabetes, obesity, and acute myocardial infarction, etc $[4,5]$. CHF's major symptoms include breath difficulty, ankle/leg swelling, and intolerance of even light exercises, etc [3], and it is usually treated with symptom relieving medications and implanted assist devices [6]. CHF is a common cardiovascular disease with more than $2 \%$ population coverage in the western countries $[7,8]$, but is rapidly becoming one of the most prevalent cardiovascular diseases in Asia, for example, case studies showed that $\mathrm{CHF}$ patients account for $0.9 \%$ of the Chinese population [9].

Even with more than 100 years of intensive studies, CHF still remains to have the worst prognoses and diagnosis treatment available as compared to the other chronic diseases, e.g. kidney failure and arthritis, and most cancers [10]. It has been reported that approximately $\sim 40 \%$ of the CHF patients die within the first year after diagnosis, and the five-year survival rate is only $25 \%$ for men and $38 \%$ for women, according to the Framingham study [11]. The high incidence of death rate and the non- availability of suitable drug treatments led to the particularly increasing interest in developing early diagnosis and cardiovascular management techniques, so that suitable preventive measures can be designed for the identification and treatment of the CHF at an early stage [12], and to improve the life span and quality after its diagnosis [13].

Two types of chronic heart failure (CHF), viz. $\mathrm{CHF}$ with reduced (CHFrEF) or preserved (CHFpEF) ejection fractions, have been widely reported [14]. Available medication strategies for both types of $\mathrm{CHF}$ remains unsatisfactory as they largely cannot stop the disease progression and restore the quality of the patient's life [15]. It has been demonstrated that acute decompensated heart failure (ADHF) develops as the disease progresses, and has been found to be strongly correlated with the recurrent hospitalization and in-hospital mortality [16]. CHFrEF may be treated with sarcoplasmic reticulum calcium ATPase 2A activators, to increase the sarcoplasmic reticulum (SR) uptake of calcium $\mathrm{Ca}^{2+}$, which is the central regulator of excitation-contraction coupling [17]. It is also essential to maintain the $\mathrm{Ca}^{2+}$ releasing channel of the heart sarcoplasmic reticulum through the activated ryanodine receptor 2 and ryanodine receptor stabilizers [18]. Novel therapies have been proposed for CHFrEF, including the growth promoting factor neuregulin-1 (NRG-1), which may facilitate the cardiac chamber differentiation, and is already in the phase II clinical testing [19]. However, some major issues need to be addressed in CHF drug develop- ment such as the organ specificity, since elevated expression of growth promoting factors in organs other than heart may also promote the tumor growth [20]. The renin-angiotensin aldosterone system (RAAS) is another ideal drug target, and multiple system inhibitors, e.g. angiotensin receptor blockers (ARBs), are clinically available [21]. CHFpEF accounts for the majority of CHF patients but no effective therapy is available as of now [22]. Two candidate therapeutic targets are the advanced glycation end products (AGEs) $[23,24]$ and the large cytoskeletal protein titin [25].

Since 2006, a number of Traditional Chinese Medicines (TCMs) were clinically tested on the CHF patients, including the warming yang strategy [26], nourishing yin strategy [27] and activating blood strategy $[27,28]$. TCM is referred to both the medical practices developed during the last few thousand years in China and the medical materials from herbal and animal substances [29]. TCM practices based on physical therapy, acupuncture and dietary treatment represent a healthy complement to the western medical practices, and already received legislations for professional practices in 43 states as of 2012. California established the first Acupuncture Board in 1975 to certify the professional acupuncturists [30]. Xinmailong was a bioactive composite extracted from periplaneta Americana, and showed reasonable protective effects against the cardiovascular injury.

In our conducted research, we performed a systematic investigation of the potential effects of the Traditional Chinese Medicine (TCM), Xinmailong, on the chronic heart failure patients, as compared with the standard treatment using five measurements, i.e. Ang_II, hs_CRP, LVESVI, LVEF and NT_proBNP. A full course of 15 days was adopted and three time points (day $0,7^{\text {th }}$ and $15^{\text {th }}$ ) were chosen for measuring the five factors, and to do a time-series analysis. We observed that both treatments were found effective for $\mathrm{CHF}$, in particular we observed that the Xinmailong showed almost double statistically significant rate as compared with the available standard treatment.

\section{Methods}

\section{Experimental protocol}

The present study was conducted to evaluate the potential therapeutic performance of the Chinese medicine, Xinmailong, on the chronic heart failure (CHF) patients, and to determine the optimal treatment course. All the recruited CHF patients, including both in- and out-patients, visited the cardiovascular department of the Xiangya Hospital in the Central South University in China between October 2009 and 
May 2011. To narrow down the investigation to the cardiovascular disorders, we excluded the patients with disorders such as liver or kidney diseases, diabetes, coagulation defects, endocrine disorders, malignant tumors, or desmosis. We also excluded the CHF patients with the history of habitual drinking or smoking, or receiving hormone replacement therapy. The control group was recruited from the healthy adults with office visits for regular physical examination in the above hospital during the same period of time.

One data profile consists of a blood test and an echocardiography. The limosis blood samples were extracted from the cubital vein before 9:00 AM, with a pre-requisite that the voluntary participants were not allowed to have a meal at least 14 hours prior to the blood extraction procedure. Blood samples were collected in an ice bathed test tube with $20 \mu \mathrm{l} 0.3 \mathrm{mM}$ EDTA, $20 \mu \mathrm{l} 0.34 \mathrm{mM}$ 8-oxychinolin, $10 \mu \mathrm{l} 0.32 \mathrm{mM}$ dimercaprol dimercaptopropanol. Then part of the blood sample was mixed evenly with the solution, before being centrifuged at $2000 \mathrm{~g}$ at $4^{\circ} \mathrm{C}$ for 15 minutes. The serum was kept for measuring the concentration of peptide hormone angiotensin II (Ang_II) by a radioimmunoassay. The serum level of the high sensitivity C-reactive protein (hs-CRP) was detected using the immune-fluorescence technique. The level of $\mathrm{N}$ terminal pro-B-type natriuretic peptide (NT-proBNP) was measured using the ReLTA Immuno-detection machine physically located in the clinical laboratory in the hospital.

An ultrasonic cardiogram (UCG, also denoted as echocardiography) was taken for each voluntary participant to calculate the left ventricular (LV) ejection fraction (LVEF) and Left Ventricular End Systolic Volume (LVESV). The body surface area (BSA) is calculated by the formula $0.0061 \times$ height $(\mathrm{cm})+0.0128 \times$ weight $(\mathrm{kg})-0.1529$ [31, 32], and the Left Ventricular End Systolic Volume Index (LVESVI) is LVESV/BSA [33].

The five parameters, i.e. Ang_II, hs_CRP, LVESVI, LVEF and NT_proBNP, were investigated because they are the major factors to measure how well the heart pumps the blood and the blood carries the oxygen.

\section{Voluntary Participants}

In our conducted research, there were 104 healthy participants in the control group, and all of them were diagnosed as normal (class I) based on the New York Heart Association (NYHA) Functional Classification system [34]. The control group was randomly split into the study group (69 participants, including 36 males and 33 females) and the test group (35 participants, including 19 males and 16 females).
The two groups of normal participants were denoted as the normal study group (sNormal) and normal test group (tNormal), and they were combined into the group Normal, respectively.

Based on the aforementioned criteria, the data of 121 participants with NYHA class II-IV diagnosis were collected for further downstream analysis. The hypothesis obtained from one participant group (study group) will be independently validated on another group of participants (test group). Only hypothesis holding for both the study and test groups is considered to be reasonable. So the 121 recruited participants were randomly split into the study group (81 participants, including 42 males and 39 females), and the test group (40 participants, including 25 males and 15 females). We further divided randomly the heart failure study group into two groups to receive different treatments. Group $s A$ has 40 patients $(17,20$ and 3 patients at NYHA class II, III and IV, respectively), and received the standard treatment with digitalis preparation, diureticum, $\beta$-Blockers, sodium nitroprusside, enteric-coated aspirin. Group $s B$ has 41 patients $(18,20$ and 2 patients at the NYHA class II, III, and IV, respectively), and received the intravenous injection of Xinmailong besides the standard treatment, respectively. Each intravenous injection uses one dose of $4 \mathrm{~mL}$ Xinmailong in $100 \mathrm{~mL} \mathrm{5 \%}$ glucose. The test group was randomly split into two subgroups $t A$ and $t B$, under standard and Xinmailong treatments. Both groups $t A$ and $t B$ consist of 5,10 and 5 patients for the NYHA class II, III and IV, respectively. The group $A$ consists of patients from $s A$ and $t A$, and group $B$ is combined from $s B$ and $t B$.

Each of the two groups of CHF patients took three measurements on day $0,7^{\text {th }}$ and $15^{\text {th }}$ of the treatment. The datasets for the sub-group $s A$ were denoted as $s A 0, s A 7$ and $s A 15$, and those for $t A$ were $t A 0, t A 7$ and $t A 15$. Similar datasets were defined for $s B$ and $t B$ as $s B 0, s B 7, s B 15, t B 0, t B 7$ and $t B 15$, respectively. All involved participants passed the skin test of Xinmailong. The present investigation was conducted in accordance to the ethical guidelines and was approved by the Ethics Committee of the Xiangya Hospital, Central South University. All participants involved in the conducted study signed the written informed consent to voluntarily participate in this study.

\section{Statistical analysis}

All the statistical analysis was conducted using the program $\mathrm{R}$ version 2.15.2 (released on October 26, 2012) [35] within the graphical user interface (GUI) of RStudio version 0.97.173 (released on November 10, 2012) [36]. All the basal characteristics of the three groups of samples were summarized as Mean \pm Stdev, 
where Mean is the averaged value across the group and Stdev is the observed standard deviation across the group. We further applied independent-sample t-test to compare the five measurements, i.e. Ang_II, hs-CRP, LVESVI, LVEF and NT-proBNP, of the groups of $C H F$ patients $(A \cup B)$ and control individuals (Normal). The NYHA class differences of the two groups of $\mathrm{CHF}$ patients receiving different treatments were investigated using the Chi-square test. The age, blood pressure and the aforementioned five measurements of the same two groups of CHF patients, i.e. $\mathrm{A}$ and $\mathrm{B}$, were compared using the independent-sample t-test. All the following statistical procedures were implemented on the five measured values, i.e. Ang_II, hs-CRP, LVESVI, LVEF and NT-proBNP. The differences between the two groups with different treatments $(A$ and $B$ ) were compared by the paired-sample t-test. We applied independent-sample t-test to compare the differences between the control group (Normal) and the two CHF patient groups $(A$ and $B)$, respectively. All the statistical correlations were considered significant if smaller than the $P$-value of 0.05 .

\section{Results and Discussion}

\section{Basal characteristics of the sample groups}

The basal characteristics of the measurements of all the participants are briefly discussed here. Firstly, we summarize the age of the studied groups Normal and $A \cup B$, which are $57.90 \pm 5.49$ and $58.56 \pm 5.96$, respectively. According to the null hypothesis that the distributions of ages in the two groups do not have differences, and the independent-sample t-test $P$-value to reject this null hypothesis is 0.3897 , which is far from the threshold, 0.05 . So we concluded that there is no obvious difference between the ages of the two groups. Table 1 shows slight to median differences in the diastolic (DBPs) and systolic blood pressures (SBPs) across the groups Normal and $A \cup B$, with $P$-values 3.20e-2 and 6.36e-11, respectively. We speculate this as reasonable since most of the CHF patients tend to have heart disorders. The five measurements, i.e. Ang_II, hs-CRP, LVESVI, LVEF and NT-proBNP, are major factors to describe whether a heart works well, and are supposed to behave abnormally in the $\mathrm{CHF}$ patients. In our results, this demonstrated hypothesis is well supported by the independent-sample t-test $P$-value, which is smaller than e- 111 for all the five measurements, as shown in Table 1.

In the present research, we divided the participants uniformly into two groups to receive different treatments for statistical evaluation. Except for the NYHA classes, all the other eight measurements of the two CHF patient groups $A$ and $B$ were compared with the independent-sample t-test, and the P-value was calculated in order to provide a basis for the rejection of the null hypothesis that there is no observed difference in the given measurement of groups $A$ and $B$. The NYHA classes of the two groups of CHF patients were compared using the Pearson's chi squared test for the evaluation of the null hypothesis. We observed that the P-values of all the eight measurements between groups $\mathrm{A}$ and $\mathrm{B}$ are significantly higher than the threshold P-value of 0.05 (Table 2), which allowed us to conclude that there is no differences in the nine measurements between groups $A$ and $B$.

Table I. Baseline characteristics of the study participants. SBP and DBP are the systolic and diastolic blood pressures, respectively. Row Ang_II is the concentration of peptide hormone angiotensin II, measured in pg/ml. Row hs-CRP is the serum level of the high sensitivity C-reactive protein in $\mathrm{mg} / \mathrm{l}$. The level of amino terminal pro-B-type natriuretic peptide is given in the row NT_proBNP in pg/l. The LVEF and LVESVI are the left ventricular ejection fraction, and the left Ventricular End Systolic Volume Index, respectively. The $P$-values for all the measurements were calculated using the independent-sample t-test. The columns "Normal $(n=104)$ " and "A $\cup B(n=121)$ " represent the data for the Normal group and the combined group of $A$ and $B$.

\begin{tabular}{llll}
\hline Variable & Normal $(\mathrm{n}=104)$ & $\mathrm{A} \cup \mathrm{B}(\mathrm{n}=121)$ & $P$-value \\
\hline Age (yr old $)$ & $57.90 \pm 5.49$ & $58.56 \pm 5.96$ & $3.90 \mathrm{E}-01$ \\
SBP, mmHG & $113.39 \pm 9.58$ & $104.14 \pm 10.62$ & $6.36 \mathrm{E}-11$ \\
DBP, mmHG & $73.29 \pm 6.22$ & $75.47 \pm 8.88$ & $3.20 \mathrm{E}-02$ \\
Ang_II & $31.81 \pm 5.06$ & $94.15 \pm 6.12$ & $3.07 \mathrm{E}-170$ \\
hs_CRP & $2.10 \pm 0.57$ & $8.05 \pm 1.04$ & $4.35 \mathrm{E}-118$ \\
NT_proBNP & $31.02 \pm 3.86$ & $54.32 \pm 3.90$ & $2.00 \mathrm{E}-141$ \\
LVEF & $59.38 \pm 3.07$ & $35.77 \pm 3.10$ & $1.36 \mathrm{E}-133$ \\
LVESVI & $104.11 \pm 9.29$ & $4313.34 \pm 292.42$ & $2.66 \mathrm{E}-112$ \\
\hline
\end{tabular}

Table 2. Comparison between groups $A$ and $B$ before treatments. The variable NYHA is the class data of the NYHA classification of the CHF patients, and the class II, III and IV were converted to 2,3 and 4 , respectively, in order to calculate the averaged value and the standard deviation. The categorized NYHA classes were tested for the group difference using the Pearson's chi squared test [44]. The other eight measurements have the same meaning as in Table I, and their $P$-values were calculated using the independent-sample t-test.

\begin{tabular}{llll}
\hline Variable & $\mathrm{A}(\mathrm{n}=60)$ & $\mathrm{B}(\mathrm{n}=61)$ & $P$-value \\
\hline NYHA & $2.77 \pm 0.67$ & $2.75 \pm 0.67$ & $9.93 \mathrm{E}-01$ \\
Age $(\mathrm{yr}$ old $)$ & $58.37 \pm 5.88$ & $58.75 \pm 6.07$ & $7.22 \mathrm{E}-01$ \\
SBP $(\mathrm{mmHG})$ & $104.72 \pm 10.93$ & $103.57 \pm 10.36$ & $5.56 \mathrm{E}-01$ \\
DBP $(\mathrm{mmHG})$ & $75.68 \pm 9.05$ & $75.26 \pm 8.77$ & $7.95 \mathrm{E}-01$ \\
Ang_II $(\mathrm{pg} / \mathrm{ml})$ & $94.25 \pm 6.02$ & $94.05 \pm 6.26$ & $8.58 \mathrm{E}-01$ \\
hs_CRP $(\mathrm{mg} / \mathrm{l})$ & $8.07 \pm 1.04$ & $8.02 \pm 1.04$ & $7.79 \mathrm{E}-01$ \\
LVESVI & $54.43 \pm 4.05$ & $54.21 \pm 3.78$ & $7.58 \mathrm{E}-01$ \\
LVEF & $35.73 \pm 3.00$ & $35.80 \pm 3.21$ & $9.02 \mathrm{E}-01$ \\
NT_proBNP $(\mathrm{pg} / \mathrm{l})$ & $4312.40 \pm 292.66$ & $4314.26 \pm 294.60$ & $9.72 \mathrm{E}-01$ \\
\hline
\end{tabular}




\section{Both the standard and Xinmailong treatments are effective}

In the comparative assessment of the Xinmailong and the currently available standard treatment, we further observed that improvement in all the five measurements of the $\mathrm{CHF}$ patient group $A$ after the standard treatments, as shown in Table 3. Angiotensin II (Ang_II) is a peptide hormone that constricts arteries and veins in the heart and therefore increases the blood pressure [37]. It has been observed that the activity of the Angiotensin II (Ang_II) in serum level is usually increased in the CHF and hypertension patients [38]. We observed that both the datasets $s A$ and $t A$ have decreased serum levels of Ang_II after seven days of the standard treatment, which is well supported by strong P-values $2.67 \mathrm{e}-12$ and $5.39 \mathrm{e}-6$ to reject the null hypothesis that the Ang_II observed level was the same after the treatment. We clearly observed a further decrease in the Ang_II levels after one more week of treatment, and the much higher decreases are supported by the P-values $1.73 \mathrm{e}-33$ and $5.01 \mathrm{e}-12$, respectively. High sensitivity C-reactive protein
(hs_CRP) is a protein whose serum level is positively correlated with the inflammation in the body, and amino terminal pro-B-type natriuretic peptide (NT_proBNP) is the N-termini of the brain natriuretic peptide whose serum level is positively associated with the CHF severeness [39]. The serum levels of both molecules are used as markers to describe how severe the cardiac risk is [39]. The continuous decreases in the two molecular levels after the standard treatment in days 7 and 15 suggest that the treatment works reasonably well on CHF, as shown in Table 3. The left ventricular (LV) ejection fraction (LVEF) is the volumetric percentage of blood pumped out of the left ventricular in one heart beat, and the reduced LVEF is associated with the poorer CHF prognoses [40, 41]. And the Left Ventricular End Systolic Volume Index (LVESVI) is calculated by the ratio between the left ventricular (LV) end-systolic volume and the body surface area, which is positively associated with the $\mathrm{CHF}$ prognoses [42]. The effectiveness of the standard treatment is further supported by the significantly increased LVEF and decreased LVESVI.

Table 3. Differences of the four CHF patient groups, $s A, t A, s B$ and $t B$, at day 0 compared with days 7 and $I 5$, respectively. Column Variable gives the names of the five measurements. The data of the five measurements for the four patient groups are given in the second, third and fifth columns, respectively. The fourth column is the list of $P$-values between days 0 and 7 for the four patient groups, and the sixth column gives the P-values calculated between days 0 and I5. The P-values in this table were calculated using the paired-sample t-test [45].

\begin{tabular}{|c|c|c|c|c|c|}
\hline Variable & sA0 & sA7 & sA0 vs sA7 & sA15 & sA0 vs sA15 \\
\hline Ang_II (pg/ml) & $94.83 \pm 6.13$ & $93.40 \pm 6.12$ & 2.67E-12 & $70.62 \pm 5.59$ & $1.73 \mathrm{E}-33$ \\
\hline hs_CRP (mg/l) & $8.11 \pm 1.04$ & $7.91 \pm 1.03$ & $1.42 \mathrm{E}-03$ & $5.77 \pm 0.94$ & $1.33 \mathrm{E}-34$ \\
\hline LVESVI & $54.27 \pm 4.09$ & $53.25 \pm 3.81$ & $6.95 \mathrm{E}-10$ & $47.08 \pm 3.80$ & $3.08 \mathrm{E}-30$ \\
\hline LVEF & $36.85 \pm 2.78$ & $37.62 \pm 2.68$ & 2.32E-04 & $41.62 \pm 2.38$ & 4.43E-29 \\
\hline NT_proBNP (pg/l) & $4318.57 \pm 298.86$ & $4103.65 \pm 419.12$ & $5.96 \mathrm{E}-06$ & $3111.80 \pm 409.03$ & $1.24 \mathrm{E}-31$ \\
\hline Variable & tA0 & tA7 & tA0 vs tA7 & tA15 & tA0 vs tA15 \\
\hline Ang_II (pg/ml) & $93.10 \pm 5.75$ & $92.00 \pm 5.80$ & $5.39 \mathrm{E}-06$ & $71.10 \pm 8.37$ & $5.01 \mathrm{E}-12$ \\
\hline hs_CRP (mg/l) & $8.00 \pm 1.07$ & $7.82 \pm 1.06$ & $1.47 \mathrm{E}-04$ & $5.54 \pm 0.98$ & $4.08 \mathrm{E}-19$ \\
\hline LVESVI & $54.75 \pm 4.05$ & $53.60 \pm 3.99$ & $4.51 \mathrm{E}-06$ & $47.40 \pm 3.97$ & 8.63E-17 \\
\hline LVEF & $33.50 \pm 2.04$ & $34.30 \pm 2.41$ & $5.80 \mathrm{E}-05$ & $38.60 \pm 2.82$ & 2.17E-16 \\
\hline NT_proBNP (pg/l) & $4300.05 \pm 287.04$ & $3211.80 \pm 295.34$ & $1.92 \mathrm{E}-20$ & $2149.60 \pm 259.82$ & 3.27E-15 \\
\hline Variable & sBo & sB7 & sB0 vs sB7 & sB15 & sB0 vs sB15 \\
\hline Ang_II (pg/ml) & $94.56 \pm 6.39$ & $93.34 \pm 6.48$ & $5.50 \mathrm{E}-11$ & $47.51 \pm 6.98$ & $9.63 \mathrm{E}-38$ \\
\hline hs_CRP (mg/l) & $8.07 \pm 1.04$ & $7.66 \pm 1.04$ & $2.16 \mathrm{E}-11$ & $3.11 \pm 0.63$ & $1.15 \mathrm{E}-33$ \\
\hline LVESVI & $53.95 \pm 3.85$ & $52.78 \pm 3.81$ & 6.04E-14 & $40.56 \pm 3.27$ & 8.10E-35 \\
\hline LVEF & $36.90 \pm 3.09$ & $37.54 \pm 3.09$ & $8.64 \mathrm{E}-07$ & $46.37 \pm 2.46$ & $3.74 \mathrm{E}-25$ \\
\hline NT_proBNP (pg/l) & $4321.17 \pm 301.67$ & $3935.17 \pm 401.83$ & $8.22 \mathrm{E}-17$ & $2065.41 \pm 371.46$ & 7.89E-37 \\
\hline Variable & tBo & tB7 & tB0 vs tB7 & tB15 & tB0 vs tB15 \\
\hline Ang_II (pg/ml) & $93.00 \pm 6.01$ & $91.90 \pm 5.97$ & $1.99 \mathrm{E}-04$ & $48.00 \pm 8.57$ & $6.23 \mathrm{E}-16$ \\
\hline hs_CRP (mg/l) & $7.93 \pm 1.06$ & $7.77 \pm 1.00$ & $4.88 \mathrm{E}-04$ & $3.26 \pm 0.89$ & $1.87 \mathrm{E}-11$ \\
\hline LVESVI & $54.75 \pm 3.65$ & $53.25 \pm 3.39$ & 3.92E-07 & $40.85 \pm 3.77$ & $9.82 \mathrm{E}-18$ \\
\hline LVEF & $33.55 \pm 2.14$ & $34.45 \pm 2.46$ & $2.98 \mathrm{E}-04$ & $42.55 \pm 2.87$ & 2.97E-13 \\
\hline NT_proBNP (pg/l) & $4300.10 \pm 286.65$ & $3210.80 \pm 294.84$ & $1.70 \mathrm{E}-20$ & $1390.60 \pm 238.24$ & $9.96 \mathrm{E}-20$ \\
\hline
\end{tabular}


The Xinmailong treatment seems to facilitate greater improvements on the CHF symptoms. Xinmailong is a composite extract made from periplaneta americana, and its effective constituent was shown to have significant protection of cardiovascular cells from the hypoxia-ischemic myocardial injury [43]. Its effective constituents still remain unknown. Firstly, Table 3 suggests that the Xinmailong treatment also effectively soothes the CHF symptoms, e.g. blood pressure (Ang_II), inflammation levels (hs_CRP), and heart physiological functions (LVEF and LVESVI). Secondly, the Xinmailong treatment induces the slightly greater decreases for all the measurements except for LVEF than the standard treatment by comparing sA7 vs sB7, and tA7 vs tB7. After 15 days, the Xinmailong treatment induces much better improvements in the four measurements than the standard treatment by comparing sA15 vs sB15, and tA15 vs tB15, and the P-values also support the significances. The Xinmailong treatment induces slightly worse improvement in the measurement LVEF compared with the standard treatment, but LVEF is known to be low in the CHF prediction sensitivity [42]. LVESVI was demonstrated to be a better CHF prognoses marker, with accurate predictions even in CHF patients with normal LVEF values [42].

\section{A full course of 15 days is necessary for both treatments}

We further investigated the treatment effectiveness by comparing the five measurements in the dataset Normal with those in the two CHF patient datasets $A$ and $B$, respectively. The reduced difference of a measurement of a CHF patient dataset compared with the normal dataset is considered as an improvement for this measurement, and the P-value calculated by the independent-sample t-test shows how significant the two datasets are different to each other. For the standard treatment, we firstly compared the two study participant groups, sNormal and $s A$. On day 0 of the treatment, $s A 0$ is significantly different from sNormal in all the five measurements, with P-values 7.31e-61, 2.04e-36, 1.66e-44, 8.00e-54 and 9.77e-47 for Ang_II, hs_CRP, LVESVI, LVEF and NT_proBNP, respectively. After 7 days of the standard treatment, the CHF patients in the dataset $s A 7$ have slightly improvements in the five measurements, i.e. increase for LVEF and decrease for the other four. But the P-values of the two calculations "sA0 vs $\mathrm{sN}^{\text {" }}$ and "sA7 vs sN" do not support that LVESVI and LVEF were significantly improved.

We also calculated the P-values of LVESVI and LVEF between the two datasets sA0 and sA7, and they got 0.25 and 0.21 in P-values using the inde- pendent-sample t-test, respectively. The data suggests that although Table 3 shows the significant improvements of LVESVI and LVEF for each CHF patient in column "sA0 vs sA7" with the paired-sample t-test $P$-values $6.95 \mathrm{e}-10$ and $2.32 \mathrm{e}-4$ (Table 3 ), but the two measurements after 7 days of treatment are still within the range of the CHF patients without treatment. All the five measurements show further improvements after day $15^{\text {th }}$ of the treatment, with significantly increased P-values. The test datasets tA0, tA7 and tA15 show similar improvements in all the five measurements with prolonged treatment period, as shown in Table 4. Although Table 3 suggests that the five measurements of individual CHF patients were significantly improved for the comparisons " $\mathrm{tA} 0$ vs tA7" and "tA0 vs tA15", the group-wise comparisons between the dataset tA0 and tA7 only support the improvement significance of NT_proBNP with $P$-value $2.74 \mathrm{e}-14(<0.05)$. But all the five measurements were significantly improved after 15 days of the standard treatment, with the P-values $<0.05$ calculated by using both paired-sample t-test (Table 3 ) and independent-sample t-test (Table 4).

The Xinmailong treatment also showed greater improvements in the five measurements, which might be facilitated with longer treatment period, and we further observed that the five measurements were only slightly improved during the middle (day $7^{\text {th }}$ ) of the Xinmailong treatment, as show in Table 3 and Table 4.

\section{Xinmailong works better than the standard treatment}

Initially, we observed that both Xinmailong and standard treatments showed similar improvements on day 7, but Xinmailong showed significantly better enhancement than the standard treatment after a full course of 15 days. Both Table 3 and Table 4 show the differences of the improvements on days 7 and 15 for the two treatments, and we investigated the statistical significance of the differences. Figure 1 (a) shows how Ang_II was improved during the course of 15 days for the two treatments. As similarly in Table 3 and Table 4 , the Xinmailong treatment doesn't show big difference on day 7 compared with the standard treatment in the study datasets $s A 7$ and $s B 7$ with $P$-value $9.67 \mathrm{e}-1$ in Table 5. Both treatments showed significant improvements of the serum Ang_II levels after 15 days of treatments, and Xinmailong showed greater decrease of Ang_II in the serum than the standard treatment with P-value 7.97e-27 ("sA15 vs sB15" in Table 5). The same differences were also observed in the test datasets tA7, tB7, tA15 and tB15 in Table 5. 
The other four measurements also supported this observation.

Figure 1 illustrates that Xinmailong significantly restored the five measurements toward the healthy status after 15 days of treatment, and almost doubled the improvements compared with the standard treatment. We tested the hypothesis on the study datasets that the Xinmailong's improvement on Ang_II, i.e. Ang_II(sB15)-Ang_II(sB0), doubles the standard treatment's improvement, i.e. $2 \times[$ Ang_II(sA15)-Ang_ II(sA0)]. The P-value 3.31e-1 in Table 5 cannot reject the hypothesis. And the investigation on the test datasets also supports that Xinmailong's improvement on Ang_II doubles that of the standard treatment, with rejecting $P$-value $7.71 \mathrm{e}-1$. The other four measurements support this hypothesis on both study and test datasets, except for the test datasets of LVEF and NT_proBNP. The test dataset of LVEF gives a marginal $P$-value $4.04 \mathrm{e}-2<0.05$ to reject the hypothesis, and that of NT_proBNP rejects the hypothesis convincingly with $P$-value $1.45 \mathrm{e}-13$, as shown in Table 5 . We may judge from Figure 1 (d) and (e) that Xinmailong facilitates greater but not doubled improvements on LVEF and NT_proBNP on both study and test datasets than the standard treatment. A further investigation for the underlining mechanism of Xinmailong treatment and a better treatment protocol may be conducted.

Table 4. Comparison of the CHF patient groups on the days 0,7 and I 5 during the treatments. Column Variable gives the names of the five measurements. For each of the four datasets, $s A, t A, s B$ and $t B$, the second column $(s N$ and $t N)$ gives the averaged value and the standard deviation of the study and test Normal datasets. The $3^{\text {th }}$ and $4^{\text {th }}$ columns give the measurements of the $\mathrm{CHF}$ patients on day 0 of the treatment and their $P$-values compared with the Normal datasets. The $5^{\text {th }}, 6^{\text {th }}, 7^{\text {th }}$ and $8^{\text {th }}$ columns are defined in a similar way.

\begin{tabular}{|c|c|c|c|c|c|c|c|}
\hline Variable & $\mathbf{s N}$ & sA0 & sA0 vs sN & sA7 & sA7 vs sN & sA15 & sA15 vs sN \\
\hline Ang_II (pg/ml) & $31.57 \pm 5.38$ & $94.83 \pm 6.13$ & 7.31E-61 & $93.40 \pm 6.12$ & $2.82 \mathrm{E}-60$ & $70.62 \pm 5.59$ & $1.65 \mathrm{E}-50$ \\
\hline hs_CRP (mg/l) & $2.30 \pm 0.56$ & $8.11 \pm 1.04$ & $2.04 \mathrm{E}-36$ & $7.91 \pm 1.03$ & $6.87 \mathrm{E}-36$ & $5.77 \pm 0.94$ & $2.52 \mathrm{E}-28$ \\
\hline LVESVI & $30.65 \pm 4.00$ & $54.27 \pm 4.09$ & $1.66 \mathrm{E}-44$ & $53.25 \pm 3.81$ & $4.24 \mathrm{E}-46$ & $47.08 \pm 3.80$ & $6.70 \mathrm{E}-36$ \\
\hline LVEF & $59.68 \pm 2.43$ & $36.85 \pm 2.78$ & $8.00 \mathrm{E}-54$ & $37.62 \pm 2.68$ & $1.06 \mathrm{E}-54$ & $41.62 \pm 2.38$ & 3.95E-54 \\
\hline NT_proBNP (pg/l) & $103.90 \pm 9.27$ & $4318.57 \pm 298.86$ & $9.77 \mathrm{E}-47$ & $4103.65 \pm 419.12$ & $3.79 \mathrm{E}-40$ & $3111.80 \pm 409.03$ & $8.60 \mathrm{E}-36$ \\
\hline Variable & $\mathbf{t N}$ & tA0 & tA0 vs tN & tA7 & tA7 vs tN & tA15 & tA15 vs tN \\
\hline Ang_II (pg/ml) & $32.29 \pm 4.38$ & $93.10 \pm 5.75$ & 4.68E-29 & $92.00 \pm 5.80$ & $1.47 \mathrm{E}-28$ & $71.10 \pm 8.37$ & $1.52 \mathrm{E}-16$ \\
\hline hs_CRP (mg/l) & $1.70 \pm 0.33$ & $8.00 \pm 1.07$ & $2.24 \mathrm{E}-17$ & $7.82 \pm 1.06$ & 3.17E-17 & $5.54 \pm 0.98$ & $6.78 \mathrm{E}-14$ \\
\hline LVESVI & $31.74 \pm 3.53$ & $54.75 \pm 4.05$ & $1.06 \mathrm{E}-21$ & $53.60 \pm 3.99$ & $2.87 \mathrm{E}-21$ & $47.40 \pm 3.97$ & $9.68 \mathrm{E}-17$ \\
\hline LVEF & $58.77 \pm 4.02$ & $33.50 \pm 2.04$ & $2.36 \mathrm{E}-35$ & $34.30 \pm 2.41$ & $1.50 \mathrm{E}-33$ & $38.60 \pm 2.82$ & 2.29E-27 \\
\hline NT_proBNP (pg/l) & $104.51 \pm 9.45$ & $4300.05 \pm 287.04$ & 7.51E-24 & $3211.80 \pm 295.34$ & 3.77E-21 & $2149.60 \pm 259.82$ & 8.78E-19 \\
\hline Variable & $\mathbf{s N}$ & sB0 & sB0 vs sN & sB7 & sB7 vs sN & sB15 & sB15 vs sN \\
\hline Ang_II (pg/ml) & $31.57 \pm 5.38$ & $94.56 \pm 6.39$ & $4.35 \mathrm{E}-60$ & $93.34 \pm 6.48$ & $1.01 \mathrm{E}-58$ & $47.51 \pm 6.98$ & $1.88 \mathrm{E}-19$ \\
\hline hs_CRP (mg/l) & $2.30 \pm 0.56$ & $8.07 \pm 1.04$ & $2.94 \mathrm{E}-37$ & $7.66 \pm 1.04$ & $1.06 \mathrm{E}-35$ & $3.11 \pm 0.63$ & 1.83E-09 \\
\hline LVESVI & $30.65 \pm 4.00$ & $53.95 \pm 3.85$ & $8.12 \mathrm{E}-48$ & $52.78 \pm 3.81$ & $1.63 \mathrm{E}-46$ & $40.56 \pm 3.27$ & $2.69 \mathrm{E}-25$ \\
\hline LVEF & $59.68 \pm 2.43$ & $36.90 \pm 3.09$ & $6.26 \mathrm{E}-50$ & $37.54 \pm 3.09$ & $4.45 \mathrm{E}-49$ & $46.37 \pm 2.46$ & $1.01 \mathrm{E}-43$ \\
\hline NT_proBNP (pg/l) & $103.90 \pm 9.27$ & $4321.17 \pm 301.67$ & $9.64 \mathrm{E}-48$ & $3935.17 \pm 401.83$ & 4.02E-41 & $2065.41 \pm 371.46$ & 4.70E-31 \\
\hline Variable & $\mathbf{t N}$ & tB0 & tB0 vs tN & tB7 & tB7 vs tN & tB15 & tB15 vs tN \\
\hline Ang_II (pg/ml) & $32.29 \pm 4.38$ & $93.00 \pm 6.01$ & $6.62 \mathrm{E}-28$ & $91.90 \pm 5.97$ & $8.16 \mathrm{E}-28$ & $48.00 \pm 8.57$ & 5.60E-08 \\
\hline hs_CRP (mg/l) & $1.70 \pm 0.33$ & $7.93 \pm 1.06$ & $2.18 \mathrm{E}-17$ & $7.77 \pm 1.00$ & $9.12 \mathrm{E}-18$ & $3.26 \pm 0.89$ & $1.55 \mathrm{E}-07$ \\
\hline LVESVI & $31.74 \pm 3.53$ & $54.75 \pm 3.65$ & $6.06 \mathrm{E}-24$ & $53.25 \pm 3.39$ & $1.38 \mathrm{E}-24$ & $40.85 \pm 3.77$ & $1.14 \mathrm{E}-10$ \\
\hline LVEF & $58.77 \pm 4.02$ & $33.55 \pm 2.14$ & 3.95E-35 & $34.45 \pm 2.46$ & $3.52 \mathrm{E}-33$ & $42.55 \pm 2.87$ & 7.61E-23 \\
\hline NT_proBNP (pg/l) & $104.51 \pm 9.45$ & $4300.10 \pm 286.65$ & 7.31E-24 & $3210.80 \pm 294.84$ & $3.68 \mathrm{E}-21$ & $1390.60 \pm 238.24$ & $9.79 \mathrm{E}-16$ \\
\hline
\end{tabular}

Table 5. Comparison of $P$-values calculated between the five measurements of the four datasets, i.e. " $s A 7$ vs $s B 7$ ", " $t A 7$ vs tB7", "sAI 5 vs sBI5" and "tAI 5 vs tBI5", respectively. The last two columns compared the improvements of the five measurements on day 15 for the study and test datasets of the two treatments, respectively. The $P$-value was calculated between the two datasets with the independent-sample t-test [45].

\begin{tabular}{lllllll}
\hline Variable & sA7 vs sB7 & tA7vs tB7 & sA15 vs sB15 & tA15 vs tB15 & $2^{*}(\mathrm{sA15}-\mathrm{sA0})$ vs (sB15-sB0) $2^{*}(\mathrm{tA15}-\mathrm{tA0})$ vs (tB15-tB0) \\
\hline Ang_II $(\mathrm{pg} / \mathrm{ml})$ & $9.67 \mathrm{E}-01$ & $9.57 \mathrm{E}-01$ & $7.97 \mathrm{E}-27$ & $1.78 \mathrm{E}-10$ & $3.31 \mathrm{E}-01$ & $7.71 \mathrm{E}-01$ \\
hs_CRP $(\mathrm{mg} / \mathrm{l})$ & $2.87 \mathrm{E}-01$ & $8.85 \mathrm{E}-01$ & $3.39 \mathrm{E}-23$ & $3.42 \mathrm{E}-09$ & $1.39 \mathrm{E}-01$ & $5.02 \mathrm{E}-01$ \\
LVESVI & $5.81 \mathrm{E}-01$ & $7.67 \mathrm{E}-01$ & $3.28 \mathrm{E}-12$ & $4.44 \mathrm{E}-06$ & $6.55 \mathrm{E}-02$ & $2.15 \mathrm{E}-01$ \\
LVEF & $8.91 \mathrm{E}-01$ & $8.47 \mathrm{E}-01$ & $2.22 \mathrm{E}-13$ & $8.76 \mathrm{E}-05$ & $8.80 \mathrm{E}-01$ & $4.04 \mathrm{E}-02$ \\
NT_proBNP $(\mathrm{pg} / \mathrm{l})$ & $6.87 \mathrm{E}-02$ & $9.92 \mathrm{E}-01$ & $1.82 \mathrm{E}-19$ & $1.04 \mathrm{E}-11$ & $8.26 \mathrm{E}-02$ & $1.45 \mathrm{E}-13$
\end{tabular}


(a)

(b)
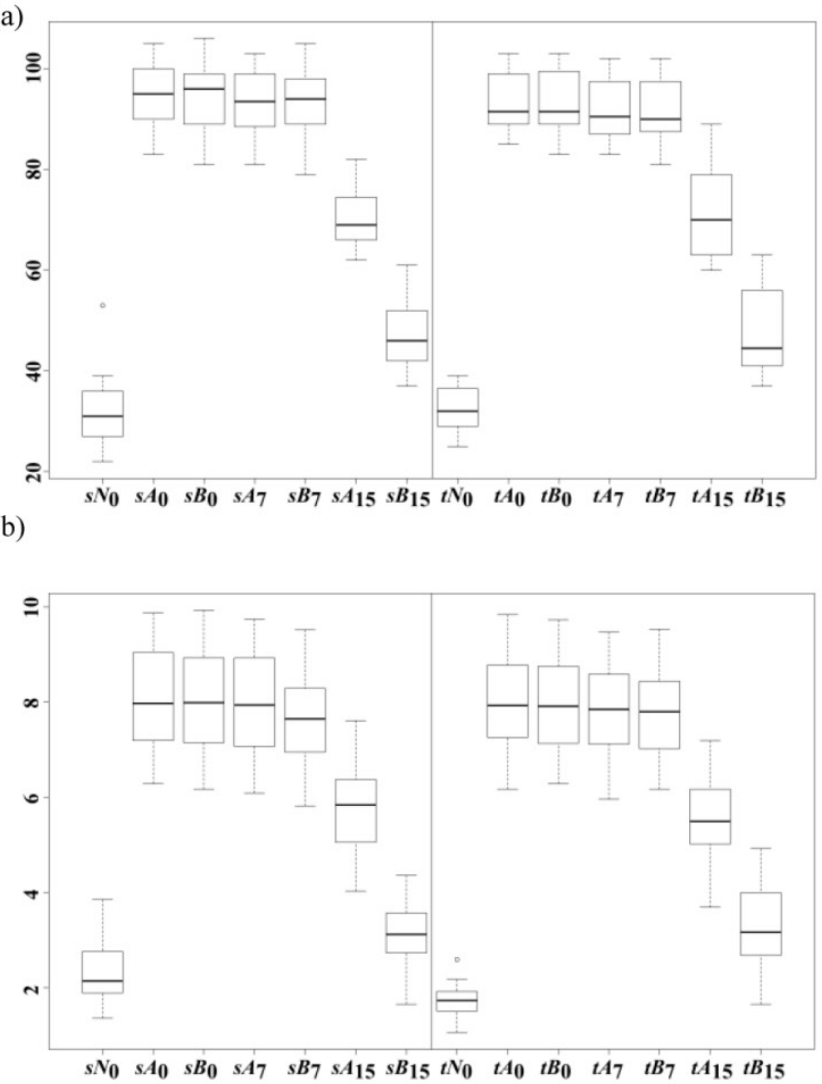

(c)

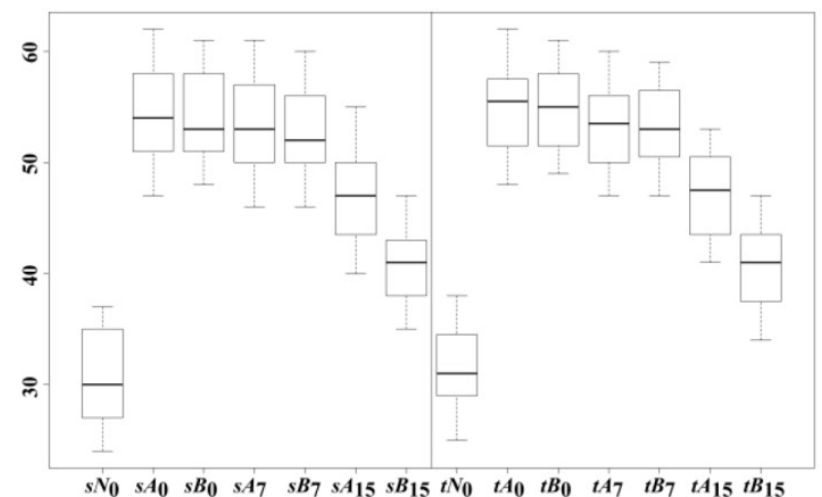

\section{Conclusion}

In our study, we conclude that Xinmailong represents a promising Traditional Chinese Medicine for the cardiovascular diseases, and is currently adopted as an optional treatment for the CHF patients in the Xiangya Hospital and a number of other large hospitals in China. The data presented in this study suggests that Xinmailong may effectively restore the multiple cardiovascular measurements to the normal levels, and achieves the doubled improvements in these measurements compared with the available (d)



(e)

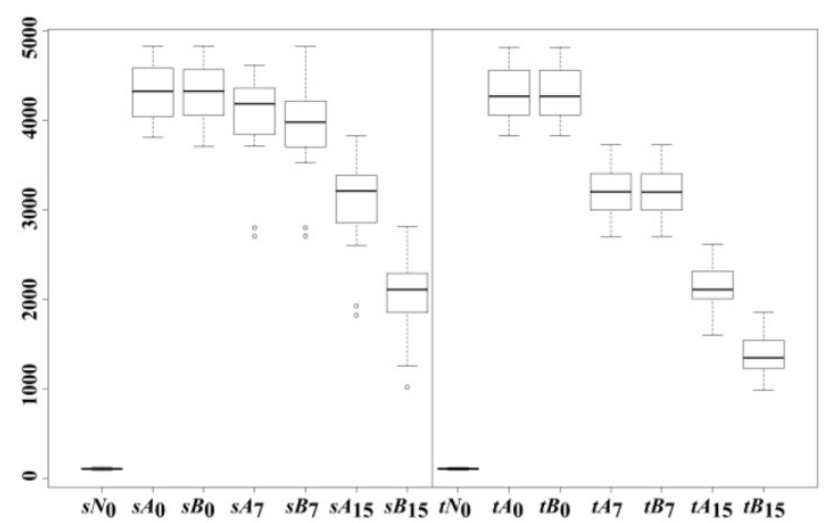

Figure I. Box plots of the five measurements of the four datasets, i.e. sA, sB, tA and tB, of (a) Ang_II, (b) hs_CRP, (c) LVESVI, (d) LVEF and (e) NT_proBNP. Each sub-figure groups the study and test groups together, respectively. A direct comparison was conducted between $s A 0$ vs $s B 0, s A 7$ vs $s B 7$, and $s A I 5$ vs $s B I 5$. So each of such pairs was plotted together. Similar setting was used for $t A 0$ vs $t B 0, t A 7$ vs $t B 7$, and $t A I 5$ vs $t B I 5$. The data suggested that the treatment $B$ is much better than $A$, and almost doubled the treatment effects after a full course of 15-day treatments. standard treatment. Subsequent studies of Xinmailong on other cardiovascular diseases are being planned, and the effective constituents of Xinmailong are being screened.

\section{Acknowledgement}

It was supported in part by National Basic Research Program of China (973 program, 2010CB732606-6), the Development Grant for the Shenzhen Key Laboratory of Low-cost Healthcare (CXB201104220026A), and NSFC Grant (31000447). 
Computing resources were partly provided by the Dawning supercomputing clusters at SIAT CAS. We would like to thank the Health Informatics Laboratory (HILab) members for their helpful discussions. This work was also partly supported by the Science and Technology Department Grant of Hunan Province (2012TT2033). We also want to thank the three anonymous reviewers for their constructive comments for this work.

\section{Competing Interests}

The authors have declared that no competing interest exists.

\section{References}

1. Dickstein K, Cohen-Solal A, Filippatos G, McMurray JJ, Ponikowski P, Poole-Wilson PA, et al. ESC guidelines for the diagnosis and treatment of acute and chronic heart failure 2008: the Task Force for the diagnosis and treatment of acute and chronic heart failure 2008 of the European Society of Cardiology. Developed in collaboration with the Heart Failure Association of the ESC (HFA) and endorsed by the European Society of Intensive Care Medicine (ESICM). Eur J Heart Fail. 2008; 10: 933-89. doi:10.1016/j.ejheart.2008.08.005.

2. Dickstein K, Cohen-Solal A, Filippatos G, McMurray JJ, Ponikowski P, Poole-Wilson PA, et al. ESC Guidelines for the diagnosis and treatment of acute and chronic heart failure 2008: the Task Force for the Diagnosis and Treatment of Acute and Chronic Heart Failure 2008 of the European Society of Cardiology. Developed in collaboration with the Heart Failure Association of the ESC (HFA) and endorsed by the European Society of Intensive Care Medicine (ESICM). Eur Heart J. 2008; 29: 2388-442. doi:10.1093/eurheartj/ehn309.

3. Butler J. An overview of chronic heart failure management. Nurs Times. 2012; 108: 16-20.

4. Ezekowitz JA, Kaul P, Bakal JA, Armstrong PW, Welsh RC, McAlister FA. Declining in-hospital mortality and increasing heart failure incidence in elderly patients with first myocardial infarction. J Am Coll Cardiol. 2009; 53: 13-20. doi:10.1016/j.jacc.2008.08.067.

5. Velagaleti RS, Pencina MJ, Murabito JM, Wang TJ, Parikh NI, D'Agostino $\mathrm{RB}$, et al. Long-term trends in the incidence of heart failure after myocardial infarction. Circulation. 2008; 118: 2057-62. doi:10.1161/CIRCULATIONAHA.108.784215.

6. Dai H, Jiang S, Li Y. Atrial activity extraction from single lead ECG recordings: Evaluation of two novel methods. Comput Biol Med. 2013; 43: 176-83.

7. de Giuli F, Khaw KT, Cowie MR, Sutton GC, Ferrari R, Poole-Wilson PA. Incidence and outcome of persons with a clinical diagnosis of heart failure in a general practice population of 696,884 in the United Kingdom. Eur J Heart Fail. 2005; 7: 295-302. doi:10.1016/j.ejheart.2004.10.017.

8. He C, Fan X, Li Y. Toward ubiquitous healthcare services with a novel efficient cloud platform. IEEE Trans Biomed Eng. 2013; 60: 230-4. doi:10.1109/TBME.2012.2222404

9. Fu S, Zhang J, Menniti-Ippolito F, Gao X, Galeotti F, Massari M, et al. Huangqi injection (a traditional Chinese patent medicine) for chronic heart failure: a systematic review. PLoS One. 2011; 6: e19604. doi:10.1371/journal.pone.0019604.

10. World_Heart_Failure_Society. Heart Failure Worldwide - Facts and Figures. 2006.

11. Stewart S, MacIntyre K, Hole DJ, Capewell S, McMurray JJ. More 'malignant' than cancer? Five-year survival following a first admission for heart failure. Eur J Heart Fail. 2001; 3: 315-22.

12. Sato Y, Fujiwara H, Takatsu Y. Biochemical markers in heart failure. J Cardiol. 2012; 59: 1-7. doi:10.1016/j.jjcc.2011.11.001.

13. Krum H, Teerlink JR. Medical therapy for chronic heart failure. Lancet. 2011; 378: 713-21. doi:10.1016/S0140-6736(11)61038-6.

14. Aronson D, Krum H. Novel therapies in acute and chronic heart failure. Pharmacol Ther. 2012; 135: 1-17. doi:10.1016/j.pharmthera.2012.03.002.

15. McMurray JJ. Clinical practice. Systolic heart failure. N Engl J Med. 2010; 362: 228-38. doi:10.1056/NEJMcp0909392.
16. Gheorghiade M, Pang PS. Acute heart failure syndromes. J Am Coll Cardiol. 2009; 53: 557-73. doi:10.1016/j.jacc.2008.10.041.

17. del Monte F, Lebeche D, Guerrero JL, Tsuji T, Doye AA, Gwathmey JK, et al. Abrogation of ventricular arrhythmias in a model of ischemia and reperfusion by targeting myocardial calcium cycling. Proc Natl Acad Sci U S A. 2004; 101: 5622-7. doi:10.1073/pnas.0305778101.

18. Shan J, Betzenhauser MJ, Kushnir A, Reiken S, Meli AC, Wronska A, et al. Role of chronic ryanodine receptor phosphorylation in heart failure and beta-adrenergic receptor blockade in mice. J Clin Invest. 2010; 120: 4375-87. doi:10.1172/JCI37649.

19. Gao R, Zhang J, Cheng L, Wu X, Dong W, Yang X, et al. A Phase II, randomized, double-blind, multicenter, based on standard therapy, placebo-controlled study of the efficacy and safety of recombinant human neuregulin-1 in patients with chronic heart failure. J Am Coll Cardiol. 2010; 55: 1907-14. doi:10.1016/j.jacc.2009.12.044.

20. Xu Y, Li X, Zhou M. Neuregulin-1/ErbB signaling: a druggable target for treating heart failure. Curr Opin Pharmacol. 2009; 9: 214-9. doi:10.1016/j.coph.2008.11.004.

21. Yao DK, Wang LX, Curran S, Ball P. Adherence to treatment guidelines in the pharmacological management of chronic heart failure in an Australian population. J Geriatr Cardiol. 2011; 8: 88-92. doi:10.3724/SP.J.1263.2011.00088.

22. Paulus WJ, van Ballegoij JJ. Treatment of heart failure with normal ejection fraction: an inconvenient truth! J Am Coll Cardiol. 2010; 55: 526-37. doi:10.1016/j.jacc.2009.06.067.

23. Willemsen S, Hartog JW, van Veldhuisen DJ, van der Meer P, Roze JF, Jaarsma T, et al. The role of advanced glycation end-products and their receptor on outcome in heart failure patients with preserved and reduced ejection fraction. Am Heart J. 2012; 164: 742-9.

24. Willemsen S, Hartog JW, Heiner-Fokkema MR, van Veldhuisen DJ, Voors AA. Advanced glycation end-products, a pathophysiological pathway in the cardiorenal syndrome. Heart Fail Rev. 2012; 17: 221-8. doi:10.1007/s10741-010-9225-z.

25. Kass DA, Bronzwaer JG, Paulus WJ. What mechanisms underlie diastolic dysfunction in heart failure? Circ Res. 2004; 94: 1533-42. doi:10.1161/01.RES.0000129254.25507.d6.

26. Chen CX, Gao JP, Wu Q, Guo J, Gu WL. [Progress in treatment of chronic heart failure in Western medicine and treatment strategies in traditional Chinese medicine]. Zhong Xi Yi Jie He Xue Bao. 2010; 8: 7-14.

27. Miao Y, Zhao WJ, Jing L. [Retrospective analysis on integrative medicinal treatment of chronic heart failure]. Zhongguo Zhong Xi Yi Jie He Za Zhi. 2008; 28: 406-9.

28. Jing L, Gao ZY. [Retrospective analysis on Chinese medicinal treatment on 302 chronic heart failure patients of coronary heart disease and diabetes]. Zhongguo Zhong Yao Za Zhi. 2008; 33: 2253-6.

29. NIH_NCCAM. Traditional Chinese Medicine: An Introduction. http://nccamnihgov/health/whatiscam/chinesemedhtm. 2010.

30. California_Acupuncture_Board. http://www.acupuncture.ca.gov/about_us/history.shtml. 1975.

31. Wang M, Lang X, Cui S, Fei K, Zou L, Cao J, et al. Clinical application of pharmacogenetic-based warfarin-dosing algorithm in patients of han nationality after rheumatic valve replacement: a randomized and controlled trial. Int J Med Sci. 2012; 9: 472-9.

32. Fang Z, Zhou L, Bao Y, Ding W, Shi H, Luo X, et al. Association of NT-proBNP and multiple biomarkers with severity of angiographic coronary artery disease in diabetic and pre-diabetic Chinese patients. PLoS One. 2011; 6: e22563.

33. Ring M, Persson H, Mejhert M, Edner M. Post-systolic motion in patients with heart failure--a marker of left ventricular dyssynchrony? Eur J Echocardiogr. 2007; 8: 352-9.

34. The_Criteria_Committee_of_the_New_York_Heart_Association. Nomenclature and Criteria for Diagnosis of Diseases of the Heart and Great Vessels. 9 ed. Boston, MA: Little, Brown \& Co. 1994;: 253-6.

35. R_Core_Team. R: A Language and Environment for Statistical Computing. Vienna, Austria. 2012.

36. RStudio_Company. RStudio IDE. Boston, Massachusetts, USA . 2012;

37. de Gasparo M, Catt KJ, Inagami T, Wright JW, Unger T. International union of pharmacology. XXIII. The angiotensin II receptors. Pharmacol Rev. 2000; 52: 415-72

38. Sobotka PA, Krum H, Bohm M, Francis DP, Schlaich MP. The role of renal denervation in the treatment of heart failure. Curr Cardiol Rep. 2012; 14: 285-92. doi:10.1007/s11886-012-0258-x.

39. Melin J, Rundstrom G, Peterson C, Bakker J, MacCraith BD, Read M, et al. A multiplexed point-of-care assay for C-reactive protein and N-terminal pro-brain natriuretic peptide. Anal Biochem. 2011; 409: 7-13. doi:10.1016/j.ab.2010.09.034 
40. Bhatia RS, Tu JV, Lee DS, Austin PC, Fang J, Haouzi A, et al. Outcome of heart failure with preserved ejection fraction in a population-based study. N Engl J Med. 2006; 355: 260-9. doi:10.1056/NEJMoa051530.

41. Owan TE, Hodge DO, Herges RM, Jacobsen SJ, Roger VL, Redfield MM. Trends in prevalence and outcome of heart failure with preserved ejection fraction. $\mathrm{N}$ Engl J Med. 2006; 355: 251-9. doi:10.1056/NEJMoa052256.

42. McManus DD, Shah SJ, Fabi MR, Rosen A, Whooley MA, Schiller NB. Prognostic value of left ventricular end-systolic volume index as a predictor of heart failure hospitalization in stable coronary artery disease: data from the Heart and Soul Study. J Am Soc Echocardiogr. 2009; 22: 190-7. doi:10.1016/j.echo.2008.11.005.

43. Huang $\mathrm{LX}, \mathrm{Wu} \mathrm{XH}$. [Effect of Xinmailong on hypoxia-inducible factor-1alpha expression in neonatal rats with asphyxia]. Zhongguo Dang Dai Er Ke Za Zhi. 2009; 11: 683-6. doi:1008-8830(2009)08-0683-04 [pii].

44. Lehmann HCaEL. The Use of Maximum Likelihood Estimates in $\chi 2$ Tests for Goodness of Fit. Annals of Mathematical Statistics. 1954; 25: 579-86.

45. Hazewinkel M. Student test. Encyclopedia of Mathematics. 2001. 